\title{
Ruptured Abdominal Aortic Aneurysm
}

The state of play

Michael Gawenda and Jan Brunkwall

\section{SUMMARY}

Background: Ruptured abdominal aortic aneurysm (rAAA) remains a challenging problem: 2,410 cases were treated in Germany in 2010. Ruptured abdominal aortic aneurysm should be suspected in patients over age 50 who complain of pain in the abdomen or back and in whom examination reveals a pulsatile abdominal mass. The incidence of hospitalization for rAAA is 12 per 100000 persons over age 65 per year (statistics for Germany, 2010), and rAAA carries an overall mortality of $80 \%$.

Methods: The current state of knowledge of rAAA was surveyed in a selective review of pertinent literature retrieved by an electronic search in the PubMed, Web of Science, and Cochrane Library databases with the keywords "abdominal aortic aneurysm," "ruptured," "open repair," and "endovascular." Publications in English or German up to and including March 2012 were considered, among them the Clinical Practice Guidelines of the European Society for Vascular Surgery (1).

Results and Conclusions: Recent reports show that the treatment of rAAA is still fraught with high mortality and high perioperative morbidity. Improvement is needed. It would be advisable for the care of rAAA to be centralized in specialized vascular centers implementing defined treatment pathways. Systematic screening, too, would be beneficial. An increasing number of reports suggest that endovascular treatment with stent prostheses improves outcomes; more definitive evidence on this matter will come from prospective, randomized trials that are now in progress.

\section{- Cite this as:}

Gawenda M, Brunkwall J: Ruptured abdominal aortic aneurysm-the state of play. Dtsch Arztebl Int 2012; 109(43): 727-32. D0I: 10.3238/arztebl.2012.0727
Cardiac Center, University Hospital of Cologne: Prof. Dr. med. Gawenda, Prof. Dr. med. Brunkwall $\triangle$ bdominal aortic aneurysm (AAA) is a dilatation of the aorta as it passes through the abdomen. 11697 patients with an asymptomatic, unruptured AAA (ICD-10 code I71.4) were treated in German hospitals in the year 2000; the corresponding figure for 2010 was 13586 patients, of whom 13\% were women. Over the same interval, the number of patients treated for ruptured AAA (rAAA, ICD-10 code I71.3) rose from 1899 to 2410 , of whom $19 \%$ were women. In 2010, 1251 persons died of rAAA in Germany, including 891 men and 360 women. (Source for all data: Federal Health Monitoring [Gesundheitsberichterstattung des Bundes].)

Ruptured abdominal aortic aneurysm (rAAA) is characterized by the presence of blood outside the adventitia of the dilated aortic wall. Free rupture, with egress of blood into the peritoneal cavity, is distinct from covered rupture, in which there is less blood loss because the periaortic hematoma is enclosed by retroperitoneal tissue (Figure 1).

There can also be retroperitoneal perforation into neighboring structures, including the inferior vena cava (aortocaval fistula) and the small or large intestine (aortoenteric fistula). An unruptured abdominal aortic aneurysm can produce symptoms resembling those of an rAAA, ranging from a mild feeling of tension to maximally intense pain with a feeling of impending doom; the defining difference between the two is the presence or absence of blood outside the adventitia (Figure 2).

Pain is caused by expansion of the aortic wall and bleeding into the intravascular thrombotic edge. The differential diagnosis of pain resembling that of an rAAA includes other abdominal conditions such as cholecystitis, diverticulitis, and pancreatitis.

The incidence of asymptomatic abdominal aortic aneurysm is estimated variably in the literature from 3.0 to 117.2 per 100000 persons per year; the reported incidence of rAAA varies from 1 to 21 per 100000 persons per year (2). The documented risk factors for abdominal aortic aneurysm are advanced age, male sex (sex ratio ranging from $1: 3$ to $1: 8$ ), positive family history, hypertension, cigarette smoking, and hypercholesterolemia (2).

The classic clinical triad of rAAA consists of

- pain in the abdomen and/or back,

- hypotension, and

- a pulsatile abdominal mass

in a patient over age 50 . 

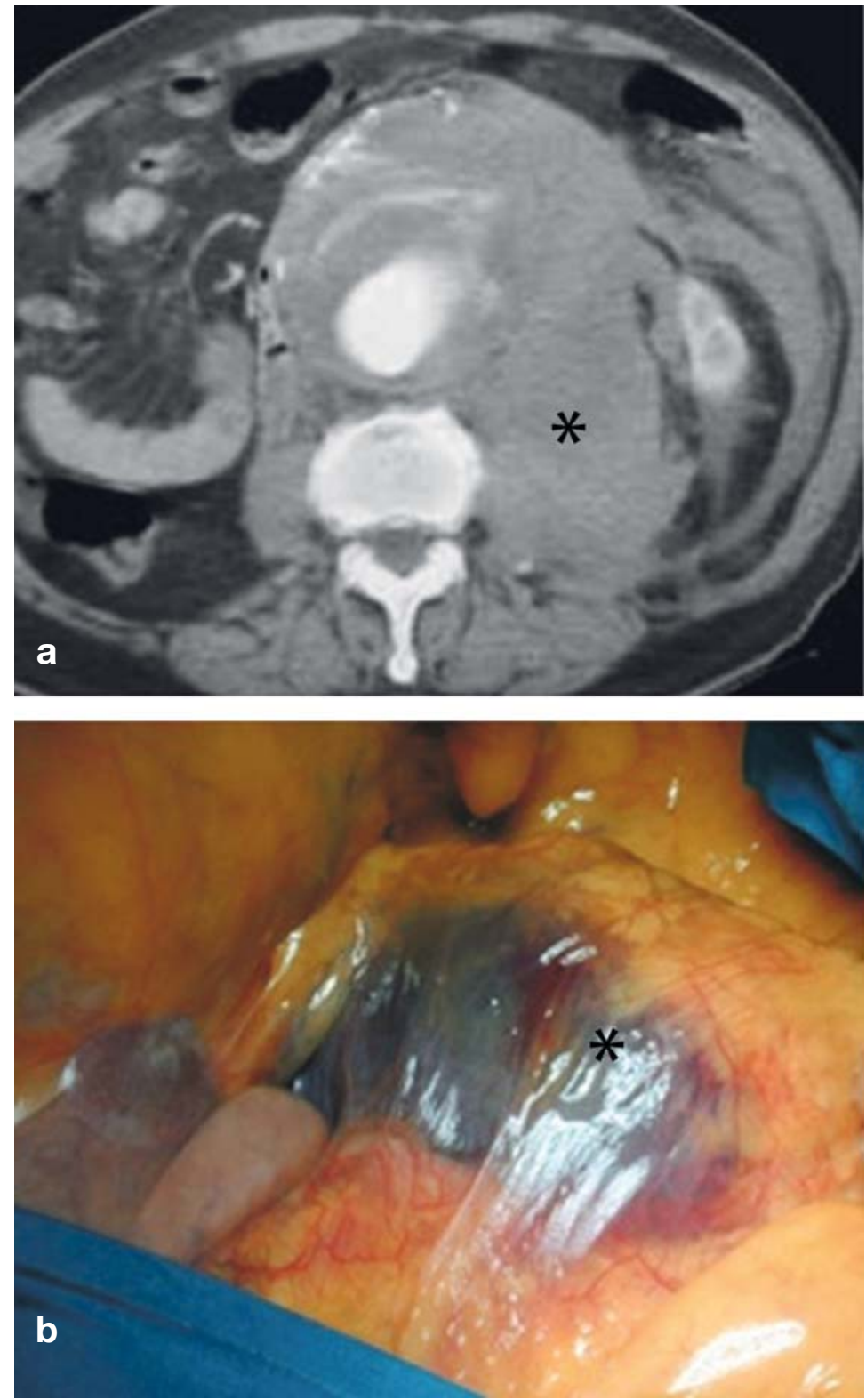

Figure 1: Covered rupture of an abdominal aortic aneurysm.

(a) Contrast-enhanced CT scan $\left({ }^{*}=\right.$ retroperitoneal hematoma),

(b) Operative field with retroperitoneal hematoma $\left.{ }^{*}\right)$.

\section{Initial treatment}

An important question in the initial treatment of patients with rAAA by the emergency medical services is whether they should be transported to the nearest hospital or to a specialized center that may be farther away (i.e., preferential referral to a high-volume hospital or high-volume vascular surgeon). Studies from the U.S.A., Canada, and the United Kingdom have shown that the latter is indeed associated with a significant lowering of mortality $(p=0.001)(3)$. In Finland, the centralized care of patients with rAAA lowered the mortality of this condition by $20 \%$ from 1996 to 2004 (4).

When a patient arrives at the hospital with a suspected rAAA, an emergency ultrasound examination is performed in the resuscitation room to confirm the diagnosis (or to determine the true cause if no rAAA is found). If possible, permissive hypotension (target systolic blood pressure, 50 to $80 \mathrm{mmHg}$; see below) should be maintained while the patient undergoes abdominal computed tomography (CT) with contrast medium. CT yields a definitive diagnosis in cases where the ultrasound findings are ambiguous (5) and provides reliable information about individual anatomy, which serve in turn as the basis for the decision whether to operate or perform an endovascular procedure. A minority of authors prefer to proceed to treatment immediately, without a CT scan, once the rAAA has been diagnosed by ultrasound; they point out that, if endovascular treatment is intended, diameters and lengths for the selection of the optimally configured stent prosthesis can be measured intraprocedurally (6).

\section{Permissive hypotension}

The concept of permissive hypotension is based on laboratory animal studies of acute hemorrhagic shock and has been successfully applied in the routine clinical management of multiple trauma (7). Animals resuscitated with permissive hypotension, rather than with restoration of normotension, survived at a significantly higher rate (8). The applicability of this concept to the treatment of rAAA has been demonstrated (9). Avoiding normal or supranormal systolic pressures is thought to make thrombus dislocation and recurrent hemorrhage ("clot popping") less likely, thereby lowering retroperitoneal blood loss (9); at the same time, infusing a lower amount of fluid lessens the harmful effects of hemodilution and the resulting hypothermia and coagulopathy (10). In a review of pertinent literature from 1990 to 2009, it was concluded that the early administration of fresh-frozen plasma (FFP) along with platelets and erythrocyte concentrate significantly lowers mortality in patients with hemorrhagic shock $(11, \mathrm{e} 1)$.

\section{Surgical management}

Permissive hypotension is maintained and the patient is transferred to the operating room, preferably a 
so-called hybrid OR that also contains the radiological equipment needed for endovascular treatment. The patient is then positioned on a warmed surface (to prevent hypothermia), and an arterial line for invasive blood pressure measurement, a nasogastric tube, a central venous catheter, and wide-bore catheters for peripheral venous access are inserted, if not already done in the resuscitation room.

If an open surgical approach has been chosen, general endotracheal anesthesia should not be initiated until the operative field has been sterilely prepped and draped, just before the skin incision. General anesthesia relaxes the muscles of the abdominal wall; sudden reduction of the abdominal pressure can convert a retroperitoneal perforation into an intraperitoneal hemorrhage, immediately causing shock (12). There are a number of different approaches and techniques for achieving the primary goal of surgery - safe, rapid, and effective crossclamping of the aorta. A prospective, randomized trial showed the transperitoneal and left extraperitoneal approaches to be equally safe and effective (13); the operating surgeon decides which to use in the individual case depending on personal experience. Some prefer to cross-clamp the aorta below the renal arteries, as in elective aneurysm surgery, while others think it advantageous to cross-clamp above the celiac artery (to avoid venous injury when a periaortic hematoma obscures surgical visibility). Some perform intraoperative hemostasis with transfemoral or transbrachial balloon occlusion, a technique borrowed from the endovascular treatment of aneurysms $(14$, e2). Intraoperative volume substitution with an autotransfusion system ("cell saver") has been shown to lessen the need for allogeneic blood transfusion (15). Mortality is also lowered by the balanced administration of FFP and erythrocyte concentrate (11).

\section{Postoperative course}

Patients with rAAA have a high postoperative morbidity (Table).

Intra- and postoperative bleeding is a major problem, exacerbated by coagulopathy. The latter can be prevented by timely surgery and by the administration of FFP, prothrombin concentrate (PPSB), and antifibrinolytic drugs (16). If a diffuse, intractable bleeding tendency nonetheless persists after the aneurysm has been repaired, intra-abdominal packing (tamponade of the retroperitoneum with closely packed, sterile abdominal compresses) may help as a last resort but confers a high risk of intra-abdominal infection in both the early and the late phases $(13 \%$ and $18 \%$, respectively) (17).

Postoperative renal failure arises in $26 \%$ to $42 \%$ of patients with $\mathrm{rAAA} ; 11 \%$ to $40 \%$ of these patients need dialysis, of whom $76 \%$ to $89 \%$ die (18). Renal failure is associated with supraceliac crossclamping, cross-clamping for more than 30 minutes, pre-existing renal dysfunction, age $>80$ and the

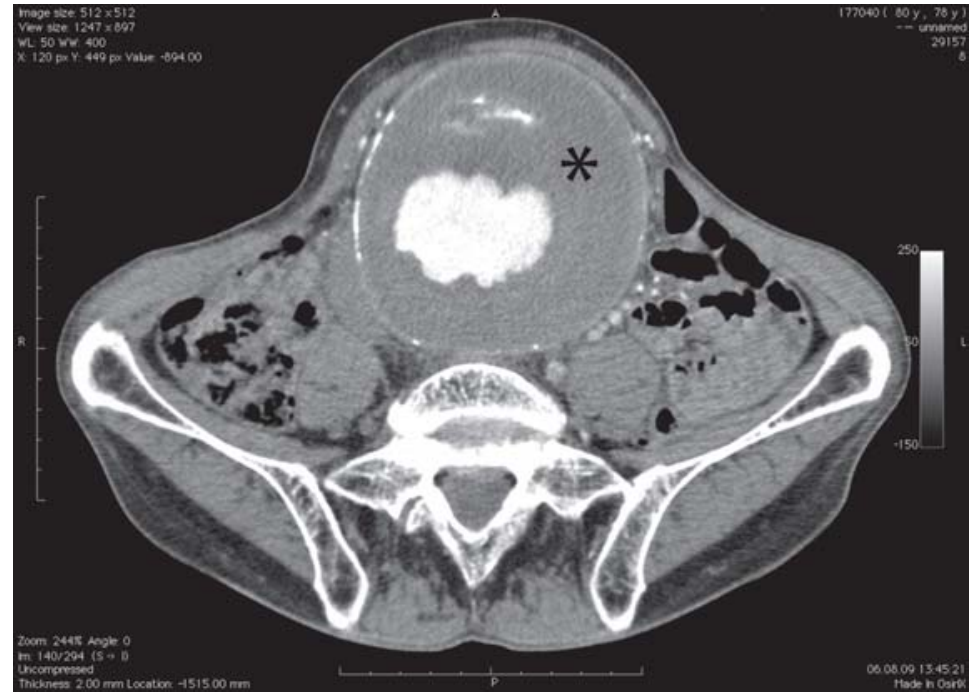

Figure 2: Contrast-enhanced CT scan of a symptomatic, unruptured abdominal aortic aneurysm $\left(^{*}=\right.$ intact aortic aneurysm with flow of contrast medium through the lumen and a curcular thrombotic edge).

central hypovolemia syndrome (“declamping shock"). The overall etiology is multifactorial, and even infrarenal cross-clamping lowers renal blood flow, with consequent lowering of the glomerular filtration rate and of urine production. Stimulation of renin production by way of the renin-angiotensin system and the sympathetic nervous system seems to play a causal role $(12,19)$. None of the putatively protective measures tested so far have been found useful, except optimization of volume status. Multiple drugs - mannitol, furosemide, fenoldopam, dopamine, popexamine, calcium-channel blockers, natriuretic peptide-have been tested without any clear finding that they lower the risk of postoperative renal failure (12).

Postoperative colonic ischemia ranges from purely mucosal involvement to transmural tissue loss; if resection is needed, the mortality is high $(73 \%-100 \%)$. This problem is more likely in the setting of perioperative hypotension, retroperitoneal hematoma, transection of the inferior mesenteric artery, and altered collateralization from the superior mesenteric artery and/or internal iliac artery. If colonic ischemia is suspected, postoperative colonoscopy can reveal it in timely fashion so that resective surgery can be performed (20). Abdominal compartment syndrome is defined as an intravesically measured abdominal pressure over $20 \mathrm{mmHg}$ together with organ dysfunction (renal failure, rising ventilatory pressure) $(21,22)$; it must be treated with abdominal decompression $(23,24)$. Some authorsseven recommend temporary abdominal wall closure (either mesh closure, or vacuum-assisted closure) for primary closure after open surgical rAAA repair (24). 
TABLE

Perioperative complications and their associated mortality

\begin{tabular}{l|c|c} 
& Frequency & Associated mortality \\
\hline Intra-/postoperative hemorrhage & $12-14 \%$ & - \\
\hline Colonic ischemia & $3-13 \%$ & $73-100 \%$ \\
\hline Respiratory insufficiency & $26-47 \%$ & $34-68 \%$ \\
\hline Renal failure & $26-42 \%$ & - \\
\hline Postoperative dialysis & $3-18 \%$ & $76-89 \%$ \\
\hline Myocardial infarction & $14-24 \%$ & $19-66 \%$ \\
\hline Cardiac arrhythmia & $19-23 \%$ & $40-48 \%$ \\
\hline Congestive heart failure & $18-21 \%$ & $39-42 \%$ \\
\hline Multi-organ failure & $59-66 \%$ & $65-71 \%$ \\
\hline Paraparesis/paraplegia & $1.1-2.3 \%$ & $45-53 \%$
\end{tabular}

About half of all persons with rAAA die before reaching a hospital, $25 \%$ of those who do reach a hospital alive die before treatment, and $40 \%$ of those who undergo surgery die; thus, the overall mortality of rAAA is roughly $80 \%(25)$. The past five decades have seen only scant improvement (lowering of perioperative mortality by $3.5 \%$ per decade) (26). Men with rAAA are about $10 \%$ more likely to survive than women (27).

\section{Risk assessment}

In theory, reliable preoperative risk assessment would let us predict how likely a patient is to survive and could be used to select surgical candidates. Expensive operative treatment could be dispensed with for patients with absolutely no chance of survival, who would instead receive humane palliative care. Although such an approach would clearly save money, it would just as clearly have ethical and legal implications that are beyond the scope of this article, which deals exclusively with medical considerations. Univariate studies have revealed a small number of risk factors associated with death after rAAA repair, which, in turn, have been incorporated into riskscoring systems. No fewer than 18 risk scores and subsequent modifications have been evaluated for their usefulness in assessing individual risk (28). The published findings are inconsistent; in any case, no score seems capable of predicting the fate of an individual patient reliably enough to permit therapeutic decision-making on the basis of the score alone. Some scores do, however, seem to be useful for comparing groups of patients in different treatment centers and for comparing the results of different types of treatment (28).

\section{Discussion}

The current treatment of patients with rAAA still has unsatisfactory results, and the question arises how it might be improved. Two approaches seem to be promising:

- Screening might shift some patients toward elective AAA repair who would otherwise have an rAAA at some later time, thereby improving the overall outcome (e3).

- Endovascular techniques that have been applied successfully in the elective treatment of asymptomatic AAA might conceivably be used with advantage in the treatment of rAAA as well.

In a recent review article in Deutsches Ärzteblatt International, Eckstein and colleagues concluded that screening for AAA would be beneficial (29). They analyzed studies from the United Kingdom, Australia, and Denmark and determined that the systematic ultrasonographic screening of men over age 65 would significantly lower the rate of AAAassociated death in the medium ( $3-5$ years) and in the long term (7-15 years), while raising the rate of elective surgery and lowering the rate of emergency surgery for AAA (29-31). The number of persons needed to screen (NNS) to prevent one death from AAA was markedly lower than the NNS for mammography (1000-2000), colonoscopy (862), or a fecal occult-blood test (808) (32).

After the initial publication of endovascular treatment techniques for abdominal aortic aneurysms (e4-e6), further studies documented their success in the elective treatment of asymptomatic AAA (33-35). Endovascular treatment was first used for rAAA in 1994; the subsequent literature contains increasing evidence that its use for rAAA may be advantageous (36). For anatomical reasons, however, only about $60 \%$ (range in published series, $18 \%-83 \%$ ) of patients with rAAA can be treated with an endovascular approach (1). Further limitations of endovascular treatment are its xdifficulty and the specialized staff and apparatus that it requires, but these can be overcome by the local implementation of a multidisciplinary, interprofessional rAAA protocol (37).

Permissive hypotension is used in the endovascular treatment of patients with rAAA just as it is in their open surgical treatment. If necessary, hemostasis can be obtained early on with a wide-lumen aortic balloon inserted either transfemorally or transbrachially. Moreover, endovascular transfemoral aneurysm repair with stent prostheses can be performed under local anesthesia. The use of uni-iliac stent prostheses seems to yield comparable results to the use of bifurcated systems (1). Multiple literature reviews have arrived at the conclusion that the endovascular method is superior to open surgery for rAAA (38), but bias probably pervades the published clinical series (e7-e11, 39): in many, patient selection (rather than randomization) seems to have taken place, and the percentage of patients given only palliative treatment 
ranged from $10 \%$ to $30 \%$. Moreover, multiple studies have shown that, for anatomical reasons, endovascular treatment is feasible for only $30 \%$ to $80 \%$ of patients. There was also marked variation from one study to another in the accompanying parameters of the treated patients: "hemodynamic stability," for example, was variably defined as a minimum systolic blood pressure of anywhere from 50 to $100 \mathrm{mmHg}$. More reliable information on this subject is likely to be obtained from the prospective, randomized trials that are currently in progress, one each in the Netherlands (AJAX), France (RCAR), and the United Kingdom (IMPROVE) (e12-e14).

Ruptured abdominal aortic aneurysm thus remains a challenging problem. Its treatment is still fraught with high mortality and high perioperative morbidity, and improvement is needed. The findings presented here imply that patients would do better if the treatment was centralized and if systematic screening was performed. Future studies will show whether endovascular treatment with stent prostheses improves outcomes.

\section{Conflict of interest statement}

The authors declare that no conflict of interest exists.

Manuscript submitted on 7 March 2012, revised version accepted on 6 June 2012.

Translated from the original German by Ethan Taub, M.D.

\section{REFERENCES}

1. Moll FL, Powell JT, Fraedrich G, et al.: Management of abdominal aortic aneurysms clinical practice guidelines of the European society for vascular surgery. Eur J Vasc Endovasc Surg 2011; 41 (Suppl1): 1-58.

2. Wilmink $A B$, Quick CR: Epidemiology and potential for prevention of abdominal aortic aneurysm. Br J Surg 1998; 85: 155-62.

3. Karthikesalingam A, Hinchliffe RJ, Loftus IM, Thompson MM, Holt PJ: Volume-outcome relationships in vascular surgery: the current status. J Endovasc Ther 2010; 17: 356-65.

\section{KEY MESSAGES}

- Ruptured abdominal aortic aneurysm remains a major medical challenge.

- The condition is often lethal, and treatment seems to yield a better outcome if it is centralized, i.e., provided in specialized centers for vascular medicine.

- The implementation of interdisciplinary/interprofessional treatment pathways enables up-to-date treatment incorporating both open surgical and endovascular approaches.

- The systematic screening of persons at risk with ultrasonography seems to lower the rupture rate.

- The role of endovascular techniques will be clearer when the results of prospective, randomized trials now in progress become available.
4. Laukontaus SJ, Aho PS, Pettila V, et al.: Decrease of mortality of ruptured abdominal aortic aneurysm after centralization and in-hospital quality improvement of vascular service. Ann Vasc Surg 2007; 21: 580-5.

5. Catalano 0, Siani A: Ruptured abdominal aortic aneurysm: categorization of sonographic findings and report of 3 new signs. J Ultrasound Med 2005; 24: 1077-83.

6. Mehta M, Kreienberg PB, Roddy SP, et al.: Ruptured abdominal aortic aneurysm: endovascular program development and results. Semin Vasc Surg 2010; 23: 206-14.

7. Dutton RP, Mackenzie CF, Scalea TM: Hypotensive resuscitation during active hemorrhage: impact on in-hospital mortality. J Trauma 2002; 52: 1141-6.

8. Mapstone J, Roberts I, Evans P: Fluid resuscitation strategies: a systematic review of animal trials. J Trauma 2003; 55: 571-89.

9. van der Vliet JA, van Aalst DL, Schultze Kool LJ, Wever JJ, Blankensteijn JD: Hypotensive hemostatis (permissive hypotension) for ruptured abdominal aortic aneurysm: are we really in control? Vascular 2007; 15: 197-200.

10. Hardy JF, De Moerloose P, Samama M: Massive transfusion and coagulopathy: pathophysiology and implications for clinical management. Can J Anaesth 2004; 51: 293-310.

11. Johansson PI, Stensballe J: Hemostatic resuscitation for massive bleeding: the paradigm of plasma and platelets-a review of the current literature. Transfusion 2010; 50: 701-10.

12. Knapp J, Bernhard M, Rauch H, Hyhlik-Durr A, Bockler D, Walther A: Anästhesiologisches Vorgehen bei elektiven Eingriffen an der Aorta. Anaesthesist 2009; 58: 1161-82.

13. Cambria RP, Brewster DC, Abbott WM, et al.: Transperitoneal versus retroperitoneal approach for aortic reconstruction: a randomized prospective study. J Vasc Surg 1990; 11: 314-24; discussion 24-5.

14. Arthurs Z, Starnes B, See C, Andersen C: Clamp before you cut: Proximal control of ruptured abdominal aortic aneurysms using endovascular balloon occlusion-Case reports. Vasc Endovascular Surg 2006; 40: 149-55.

15. Kauvar DS, Sarfati MR, Kraiss LW: Intraoperative blood product resuscitation and mortality in ruptured abdominal aortic aneurysm. J Vasc Surg 2012; 55: 688-92.

16. Mell MW, O'Neil AS, Callcut RA, et al.: Effect of early plasma transfusion on mortality in patients with ruptured abdominal aortic aneurysm. Surgery 2010; 148: 955-62.

17. van Herwaarden JA, van Vroonhoven TJ: Abdominal packing for surgically uncontrollable hemorrhage in ruptured abdominal aortic aneurysm repair. J Vasc Surg 2001; 33: 195-6.

18. Magee TR, Scott DJ, Dunkley A, et al.: Quality of life following surgery for abdominal aortic aneurysm. Br J Surg 1992; 79: 1014-6.

19. Bonventre JV: Pathophysiology of acute kidney injury: roles of potential inhibitors of inflammation. Contrib Nephrol 2007; 156: 39-46.

20. Harris LM, Faggioli GL, Fiedler R, Curl GR, Ricotta JJ: Ruptured abdominal aortic aneurysms: factors affecting mortality rates. J Vasc Surg 1991; 14: 812-8; discussion 9-20.

21. Bjorck M, Wanhainen A, Djavani K, Acosta S: The clinical importance of monitoring intra-abdominal pressure after ruptured abdominal aortic aneurysm repair. Scand J Surg 2008; 97 183-90.

22. Djavani Gidlund K, Wanhainen A, Bjorck M: Intra-abdominal hypertension and abdominal compartment syndrome after endovascular repair of ruptured abdominal aortic aneurysm. Eur J Vasc Endovasc Surg 2011; 41: 742-7.

23. Meldrum DR, Moore FA, Moore EE, Franciose RJ, Sauaia A Burch JM: Prospective characterization and selective management of the abdominal compartment syndrome. Am J Surg 1997; 174: 667-72; discussion 72-3.

24. Rasmussen TE, Hallett JW Jr, Noel AA, et al.: Early abdominal closure with mesh reduces multiple organ failure after ruptured abdominal aortic aneurysm repair: guidelines from a 10-year case-control study. J Vasc Surg 2002; 35: 246-53. 
25. Taylor LM Jr, Porter JM: Basic data related to clinical decisionmaking in abdominal aortic aneurysms. Ann Vasc Surg 1987; 1: 502-4.

26. Bown MJ, Sutton AJ, Bell PR, Sayers RD: A meta-analysis of 50 years of ruptured abdominal aortic aneurysm repair. Br J Surg 2002; 89: 714-30.

27. Mureebe L, Egorova N, McKinsey JF, Kent KC: Gender trends in the repair of ruptured abdominal aortic aneurysms and outcomes. J Vasc Surg 2010; 51: 9-13.

28. Gawenda M, Block A, Majd P, Brunkwall J: Was leisten Risiko-Scores beim rupturierten abdominalen Aortenaneurysma (rAAA)? Zentralbl Chir 2012; (in press) DOl: 10.1055/s-0031-1283885.

29. Eckstein HH, Bockler D, Flessenkamper I, Schmitz-Rixen T, Debus S, Lang W: Ultrasonographic screening for the detection of abdominal aortic aneurysms. Dtsch Arztebl Int 2009; 106: $657-63$

30. Lindholt JS, Norman P: Screening for abdominal aortic aneurysm reduces overall mortality in men. A meta-analysis of the mid- and long-term effects of screening for abdominal aortic aneurysms. Eur J Vasc Endovasc Surg 2008; 36: 167-71.

31. Takagi H, Goto SN, Matsui M, Manabe H, Umemoto T: A further meta-analysis of population-based screening for abdominal aortic aneurysm. J Vasc Surg 2010; 52: 1103-8.

32. Rembold CM: Number needed to screen: development of a statistic for disease screening. BMJ 1998; 317: 307-12.

33. EVAR (1) trial p. Endovascular aneurysm repair and outcome in patients unfit for open repair of abdominal aortic aneurysm (EVAR trial 2): randomised controlled trial. Lancet 2005; 365: 2187-92.

34. EVAR (2) trial p. Endovascular aneurysm repair versus open repair in patients with abdominal aortic aneurysm (EVAR trial 1): randomised controlled trial. Lancet 2005; 365: 2179-86.
35. Prinssen M, Buskens E, Blankensteijn JD: The Dutch Randomised Endovascular Aneurysm Management (DREAM) trial. Background, design and methods. J Cardiovasc Surg (Torino) 2002; 43: 379-84.

36. Veith FJ, Lachat M, Mayer D, et al.: Collected world and single center experience with endovascular treatment of ruptured abdominal aortic aneurysms. Ann Surg 2009; 250: 818-24.

37. Mehta M, Taggert J, Darling RC, et al.: Establishing a protocol for endovascular treatment of ruptured abdominal aortic aneurysms: outcomes of a prospective analysis. J Vasc Surg 2006; 44: 1-8.

38. Ten Bosch JA, Cuypers PW, van Sambeek M, Teijink JA: Current insights in endovascular repair of ruptured abdominal aortic aneurysms. Euro Intervention 2011; 7: 852-8.

39. Visser JJ, van Sambeek MR, Hamza TH, Hunink MG, Bosch JL: Ruptured abdominal aortic aneurysms: endovascular repair versus open surgery-systematic review. Radiology 2007; 245: $122-9$

\section{Corresponding author}

Prof. Dr. med. Michael Gawenda

Leitender Oberarzt der Klinik für Gefäßchirurgie

Herzzentrum, Uniklinik Köln

Universität zu Köln

Kerpener Str. 62

50937 Köln, Germany

michael.gawenda@uk-koeln.de 


\title{
REVIEW ARTICLE
}

\section{Ruptured Abdominal Aortic Aneurysm}

The state of play

\author{
Michael Gawenda and Jan Brunkwall
}

\section{EREFERENCES}

e1. Dries DJ: Hypotensive resuscitation. Shock 1996; 6: 311-6.

e2. Matsuda $\mathrm{H}$, Tanaka $\mathrm{Y}$, Hino $\mathrm{Y}$, et al.: Transbrachial arterial insertion of aortic occlusion balloon catheter in patients with shock from ruptured abdominal aortic aneurysm. J Vasc Surg 2003; 38 : 1293-6.

e3. Lindholt JS, Fasting U: Screening for abdominal aortic aneurysm among patients referred to vascular laboratory. Indeed feasible-but acceptable? Eur J Vasc Endovasc Surg 2008; 35: 675-6.

e4. Parodi JC, Palmaz JC, Barone HD: Transfemoral intraluminal graft implantation for abdominal aortic aneurysms. Ann Vasc Surg 1991; 5: 491-9.

e5. Volodos NL, Karpovich IP, Troyan VI, et al.: Clinical experience of the use of self-fixing synthetic prostheses for remote endoprosthetics of the thoracic and the abdominal aorta and iliac arteries through the femoral artery and as intraoperative endoprosthesis for aorta reconstruction. Vasa Suppl 1991; 33: 93-5.

e6. Volodos NL, Shekhanin VE, Karpovich IP, Troian VI, Gur'ev lu A: A self-fixing synthetic blood vessel endoprosthesis. Vestn Khir Im II Grek 1986; 137: 123-5.

e7. Harkin DW, Dillon M, Blair PH, Ellis PK, Kee F: Endovascular ruptured abdominal aortic aneurysm repair (EVRAR): a systematic review. Eur J Vasc Endovasc Surg 2007; 34: 673-81.

e8. Mastracci TM, Garrido-Olivares L, Cina CS, Clase CM: Endovascular repair of ruptured abdominal aortic aneurysms: a systematic review and meta-analysis. J Vasc Surg 2008; 47: 214-21.

e9. Rayt HS, Sutton AJ, London NJ, Sayers RD, Bown MJ: A systematic review and meta-analysis of endovascular repair (EVAR) for ruptured abdominal aortic aneurysm. Eur J Vasc Endovasc Surg 2008; 36: 536-44.

e10. Sadat U, Boyle JR, Walsh SR, Tang T, Varty K, Hayes PD: Endovascular vs open repair of acute abdominal aortic aneurysms-a systematic review and meta-analysis. J Vasc Surg 2008; 48: 227-36.

e11. Takagi H, Umemoto T: A meta-analysis of randomized and riskadjusted observational studies of endovascular versus open repair for ruptured abdominal aortic aneurysm. Vasc Endovascular Surg 2011; 45: 717-9.

e12. Amsterdam Acute Aneurysm trial: Amsterdam Acute Aneurysm trial: background, design, and methods. Vascular 2006; 14: $130-5$.

e13. Desgranges P, Kobeiter H, Castier Y, Senechal M, Majewski M, Krimi A: The Endovasculaire vs Chirurgie dans les Anevrysmes Rompus PROTOCOL trial update. J Vasc Surg 2010; 51: 267-70.

e14. Powell JT: Time to IMPROVE the management of ruptured abdominal aortic aneurysm: IMPROVE trialists. Eur J Vasc Endovasc Surg 2009; 38: 237-8. 